assessments would help demonstrate improved trainee knowledge, confidence, and disaster medicine skills.

Methods.-Diverse groups of medical university students, healthcare professionals, and community members were enrolled between 2011 and 2014 . The course consisted of an online questionnaire, didactic lectures, small-group exercises and 2 live, multipatient mass casualty incident (MCI) scenarios. When not participating as responders during an MCI, trainees served as patients, significantly increasing the "chaos" of the scenario. They also filled out team performance observations cards, greatly simplifying performance data collection.

Results.-Seven hundred and eight participants completed training between November 2011 and August 2014, including 49.9\% physicians, 31.9\% medical students, $7.2 \%$ nurses, and $11 \%$ various other healthcare professions. All participants completed the pretest and $71.9 \%$ completed the post-test, with average correct answers increasing from $39 \%$ to $60 \%$. After didactics, trainees met $73 \%$ and $96 \%$ of performance objectives for the 2 small group exercises and $68.5 \%$ and $61.1 \%$ of performance objectives for the $2 \mathrm{MCI}$ scenarios. Average trainee self-assessment of both overall knowledge and confidence with clinical disasters improved from 33/100 to 74/100 (overall knowledge) and 33/100 to 77/ 100 (overall confidence). The course assessment was completed by $34.3 \%$ of participants, $91.5 \%$ of whom highly recommended the course.

Conclusion.-Our EPT course demonstrated improved trainee knowledge, confidence, and disaster medicine skills with the help of trainees acting as responders, patients, and observers. This unique EPT curriculum may help educators with limited resources implement performance-based medical team training effectively and efficiently.

\section{A Novel Cooling Method and Comparison of Active Rewarming of Mild Hypothermia}

Mark L. Christensen, $\mathrm{DO}^{1}$; Grant S. Lipman, $\mathrm{MD}^{1}$; Dennis A. Grahn, $\mathrm{PhD}^{2}$; Kate Shea, $\mathrm{MD}^{1}$; Joseph Einhorn, $\mathrm{MD}^{1}$; Craig Heller, $\mathrm{PhD}^{2}$

${ }^{1}$ Department of Emergency Medicine, Stanford University School of Medicine, Stanford, CA, USA, ${ }^{2}$ Department of Biology, Stanford University, Stanford, CA, USA

Introduction.- No current technique has been established to effectively rewarm hypothermic victims in the prehospital environment, and testing treatments with established cooling models is suboptimal.

Objectives. - To compare the effectiveness of arteriovenous anastomosis (AVA) vs heated intravenous fluid (IVF) rewarming in hypothermic subjects. Additionally, to develop a novel method of inducing mild hypothermia.

Methods. - Eight subjects underwent 3 cooling trials each to a mean core temperature of $<35^{\circ} \mathrm{C}$ by $14^{\circ} \mathrm{C}$ water immersion for 30 minutes, followed by walking on a treadmill for 5 minutes for convective "afterdrop" cooling. Core temperatures changes $\left(\Delta\right.$ tes) and rates of cooling $\left({ }^{\circ} \mathrm{C} / \mathrm{h}\right)$ were measured by continuous esophageal measurements. Participants were then rewarmed by 1) control: shivering in a sleeping bag; 2) IVF: shivering in sleeping bag and infusion of $2 \mathrm{~L}$ normal saline warmed to $42^{\circ} \mathrm{C}$ at $77 \mathrm{~mL} / \mathrm{min}$; 3) AVA: shivering in sleeping bag and circulation of $45^{\circ} \mathrm{C}$ warmed fluid through neoprene pads affixed to the palms and soles of the feet.

Results. - Cold water immersion resulted in $0.5^{\circ} \mathrm{C} \pm 0.5 \Delta$ tes and $1^{\circ} \mathrm{C} \pm$ $0.3 \Delta$ tes with afterdrop $(P<.01)$; with an immersion cooling rate of $0.9^{\circ} \mathrm{C} / \mathrm{h} \pm$ 0.8 vs $12.6^{\circ} \mathrm{C} / \mathrm{h} \pm 3.2$ with afterdrop $(P<.001)$. After cooling, core temperatures reached a nadir of $35.0^{\circ} \mathrm{C} \pm 0.5^{\circ} \mathrm{C}$. There were no significant differences in rewarming rates between the 3 conditions (shivering: $1.3^{\circ} \mathrm{C} / \mathrm{h} \pm$ $0.7, \mathrm{R} 2=0.683$; IVF: $1.3^{\circ} \mathrm{C} / \mathrm{h} \pm 0.7, R^{2}=0.863$; and AVA: $1.4^{\circ} \mathrm{C} / \mathrm{h} \pm 0.6, R^{2}$ $=0.853 ; P=.58)$. Shivering inhibition was greater with AVA, but not significantly different $(P=.07)$.

Conclusion.-This study developed a novel and efficient model of hypothermia induction through exercise-induced convective afterdrop. Although there was not a clear benefit in either of the 2 active rewarming methods, AVA rewarming showed a trend toward greater shivering inhibition, which may be optimized by an improved interface.

Down to the Wire? Ventilation During Intubated Stretcher Helicopter Winching

John D. Hollott, MD

Hunter Retrieval Service, Newcastle, NSW, Australia
Introduction.- - The stretcher winching of intubated patients into a helicopter is an uncommon procedure. Positive pressure ventilation is required, which can be performed either manually with a self-inflating bag or automatically using a mechanical ventilator. The optimal method of ventilation has not been studied. Automatic ventilation has theoretical advantages in maintaining better oxygenation and stable arterial carbon dioxide levels, with less risk of airway disconnection. There is also likely to be better performance and situational awareness of the stretcher attendant during the winch, contributing to crew and patient safety. However, the ability to diagnose and manage airway disconnections and ventilator failure is of concern.

Objective.-To compare automatic vs manual ventilation during intubated stretcher helicopter winching.

Methods.-There were 2 phases to the trial. An initial static winch with a hoisting simulator was performed with 10 stretcher attendants, followed by a live helicopter winch at 30 feet into a Bell 412 with 5 attendants. Each attendant performed 2 separate winches with an intubated mannequin. During one winch the mannequin was ventilated with manual (bag) ventilation; an automatic ventilator was used for the other. Airway pressures were measured.

Results. - The results showed no failure of automatic ventilation. Automatic ventilation was superior to manual ventilation. Automatic ventilation allowed for better situational awareness and perceived performance in winching maneuvers. There was one airway disconnection during a manual ventilation winch into the aircraft.

Conclusions.-Automatic ventilation is superior in providing stable, reliable, uninterrupted ventilation during simulated helicopter winching. The risk of ventilator failure and disconnection is low. Medical retrieval organizations should develop standard operating procedures, equipment, and training to facilitate automatic ventilation during helicopter winching.

\section{Prehospital Interventions During Mass Casualty Events in Afghanistan: A Case Series}

Steven G. Schauer, DO, RDMS ${ }^{1}$; Derek Brown, MD $^{1}$; Michael D. April, $\mathrm{MD}, \mathrm{PhD}^{2}$; Erica Simon, $\mathrm{MD}^{2}$; Joseph Maddry, $\mathrm{MD}^{3}$; Robert Carter, $\mathrm{PhD}^{1}$; Robert Delorenzo, MD, MSM, $\mathrm{MSCl}^{4}$ ${ }^{1}$ US Army Institute for Surgical Research, JBSA Fort Sam Houston, TX, USA, ${ }^{2}$ San Antonio Military Medical Center, JBSA Fort Sam Houston, TX, USA, ${ }^{3}$ US Air Force 59th Medical Detachment Wing, JBSA Fort Sam Houston, TX, USA, ${ }^{4}$ University of Texas Health Sciences Center at San Antonio, San Antonio, TX, USA

Objective.-Mass casualty (MASCAL) events are known to occur in the combat setting. Data from the Joint Theater wars, specific to MASCAL events, are very limited at this time. We seek to provide preliminary data for developing prehospital planning and guidelines.

Methods.-Cases were identified using the Department of Defense Trauma Registry and the Prehospital Trauma Registry. These cases were identified during the course of an approved quality assurance analysis. Cases that were designated as or associated with MASCAL events were included.

Results.-Fifty cases were identified during the course of this project. The majority $(74 \%, \mathrm{n}=37)$ of those injured were battle injuries. Explosives were the most common cause of injuries. There was a wide range of vital signs. Tourniquet placement $(20 \%)$ and pressure dressings $(19 \%)$ were the most common interventions, followed by analgesia administration (15\%). Oral transmucosal fentanyl citrate was the most common parenteral analgesic drug administered. Most were evacuated as "routine." Follow-up data were available for 36 of the subjects, and $97 \%$ were discharged alive.

Conclusions.- - In this case series, the most common prehospital interventions were hemorrhage control followed by medication administration. This preliminary data set may help develop prehospital guidelines and planning. Larger data sets are need to validate these findings, which may have limited utility in the noncombat setting.

Core Content for Wilderness Medicine Training: Development of a Wilderness Medicine Track within an Emergency Medicine Residency

Jonathan Drew, MD ${ }^{1}$; Nicole Battaglioli, MD, FAWM ${ }^{2}$; Walter A. Schrading, MD, FACEP, FAWM ${ }^{3}$

${ }^{1}$ Indiana University Department of Emergency Medicine Residency Training Program, Indianapolis, IN, USA, ${ }^{2}$ WellSpan York Hospital 